\title{
Driver behaviour during haptic and visual secondary tasks
}

\author{
Annie Rydström \\ Dept. of Human Work Sciences \\ Luleå University of Technology \\ SE-971 87 Luleå, Sweden \\ annryd@Itu.se
}

\author{
Camilla Grane \\ Dept. of Human Work Sciences \\ Luleå University of Technology \\ SE-971 87 Luleå, Sweden \\ camgra@Itu.se
}

\author{
Peter Bengtsson \\ Dept. of Human Work Sciences \\ Luleå University of Technology \\ SE-971 87 Luleå, Sweden \\ pebn@Itu.se
}

\begin{abstract}
There has been an increasing interest for in-vehicle interfaces that make use of haptic information. A simulator study was conducted to investigate whether haptic information can facilitate the interaction with an interface while driving. The conceptual in-car interface consisted of a visual menu of four textures displayed on a screen and corresponding haptic information displayed through the interaction device - a rotary device. The experimental conditions included either visual or haptic or both visual and haptic information. One advantage of the condition including only haptic information was that the participants' eyes remained on the road during the interaction. However, since the haptic interaction necessitated serial processing, the experimental task took longer when using only haptic information. Therefore the participants seem to have relied more on the visual information when it was available. The degradation in driving performance and mental workload assessment did not differ between the conditions.
\end{abstract}

\section{Categories and Subject Descriptors}

H.5.2 [Information Interfaces and Presentation]: User Interfaces - haptic I/O

\section{General Term}

Human Factors

\section{Keywords}

In-car interfaces; Haptic information; Driver distraction

\section{INTRODUCTION}

The information input needed for driving a car is predominantly visual [25] and the major output is generally manual by the hands (steering wheel and gear shift) and by the feet (accelerator, break pedal and clutch). Visual in-vehicle displays require the eyes to be taken off the road and manual controls often require the hands to be taken off the steering wheel. While gaze has to be moved from the road to an in-vehicle display to gather detailed information, the two hands can perform different manual tasks simultaneously [31]. According to Wierwille [31] it is easier to

Copyright held by authors

AutomotiveUI'09, September 21-22, 2009, Essen, Germany ACM 978-1-60558-571-0/09/009 time-share driving and manual in-vehicle tasks than driving and visual in-vehicle tasks, as long as large corrections of the steering wheel that require the use of both hands are not needed. It is a challenge for vehicle manufacturers to design in-vehicle systems that can be operated safely while driving. It has been suggested that haptic cues available through the interaction devices may have the potential to make the interaction with in-car interfaces safer since the interaction may be less visually demanding $[3,30]$.

Humans can passively perceive vibrations, applied forces and motions. In the automotive domain the use of vibrotactile stimuli has been most investigated and has been shown to be useful as warnings $[11,16]$, as well as to provide navigation information [27]. Humans can actively, through hand movements, perceive shapes, sizes, textures and locations. The perceptions resulting from these active movements are sequential and form what is called haptic perception [9]. Haptic interaction implies the ability to both sense and manipulate an interface [12]. Hence, haptic cues can be used to create haptically discriminable buttons and switches [19]. Haptic cues can also be provided through one single interaction device, which can change its mechanical properties just as a graphic display can change its optical properties [10]. In the domain of desktop interaction, augmenting a visual interface with usable haptic information through the interaction device (e.g. a computer mouse), such as texture, friction, gravity and force, has been shown to result in a decrease in task completion time $[1,4]$ and decrease in workload and the number of errors made [7, 20]. Although there are in-car interfaces available on the market providing haptic cues through single multifunctional interaction devices [24], there has not yet been sufficient research on the use of haptic cues while driving [2]. In a study by Porter et al. [21] an in-car interface was designed in which the interface devices (three pods) were coded in terms of the haptic properties size, shape and location. It was shown that the number and duration of glances made to the display and controls were reduced compared to a standard interface. However, in the applied study by Porter et al. the comparison between the interfaces was not entirely systematic since the haptically coded interface and the standard interface differed in many aspects.

Studies carried out in real and simulated driving environments have shown that interaction with equipment within the car while driving cause changes in visual behaviour and driving performance. It has been shown that visual-manual tasks, such as interacting with a mobile phone or manipulating a car radio, leads to frequent and long periods of visual time off road and impaired lane-keeping performance, harmed detection ability and increased brake reaction time $[15,26,32,33]$. It has also been revealed that non-visual withdrawal of attention, for example a phone 
conversation, leads to impaired driving [15]. There are several different ways to measure drivers' visual behaviour and driving performance. One limitation of the ISO metrics glance frequency and glance duration [13] is that these measures only can be used to measure visual behaviour during visual secondary tasks. It has been shown that non-visual secondary tasks can lead to gaze concentration towards the centre of the road $[22,29]$. Hence, an alternative to glance based measures is the measure called Percent Road Centre (PRC) [29]. This measure focuses on how much time is spent looking at the centre area of the road and can be used to evaluate visual behaviour during both visual and non-visual secondary tasks. Several driving simulation software programs are available for evaluating the distraction caused by the interaction with in-vehicle systems. The simulations range from advanced ones that record numerous measures on both lateral and longitudinal performance to more hands-on ones that record one or a few measures. An applied driving simulation software currently under investigation to be an ISO standard is the Lane Change Test (LCT) [14]. In this PC simulated environment drivers are requested to change lanes while interacting with an incar interface. The perception and reaction to lane-change signs shows the driver's awareness of the environment and the lanekeeping shows the driver's ability to control the vehicle [18]. The LCT derives a single measure of driving performance - the mean deviation from a normative path. Mattes [18] showed that results from the LCT correlates with results from a high end moving base driving simulator. Since not only visual and manual, but also mental workload, leads to impaired driving [15] it is central to also consider the mental workload imposed by different secondary tasks. Mental workload can be measured by using subjective assessment techniques.

The objective of the present simulator study was to investigate whether haptic information can ease the interaction with an interface while driving. The conceptual interface used in the study provided visual or haptic or both visual and haptic information. Secondary task performance, eye movement behaviour, driving performance and subjective assessment of mental workload were measured.

\section{METHOD}

\subsection{Participants}

Forty participants (six women and 34 men) were recruited to take part in the study. The majority of the participants were students recruited via e-mail at Chalmers University of Technology. Their ages ranged from 20 to $46(M=26.8, S D=5.2)$. The criteria for participation were possession of a driving license and no need of eyeglasses (to ensure eye-tracking data of good quality). The participants were to be able to wear contact lenses if vision correction was needed.

\subsection{Equipment}

The study was conducted using a fixed base Volvo XC90 driving simulator (Fig. 1). A $140 \mathrm{~cm}$ wide and $110 \mathrm{~cm}$ high driving scene was projected on a screen approximately $200 \mathrm{~cm}$ in front of the participants. The driving simulation used was the Lane Change Test (LCT) [14]. In the LCT simulation a participant drives at a constant speed of $60 \mathrm{~km} / \mathrm{h}$ on a straight, three-lane road on which no other cars are present. Signs on both sides of the road instruct the participant to change lanes. The information appears 40 metres ahead of the sign. In the LCT, the deviation from a normative path is recorded. Consequently, late perception of signs (or missed signs), slow lane change and poor lane-keeping result in greater deviation [18]. An LCT track takes three minutes to complete, and 18 lane changes are made during a track. An LCT analysis software was used to compute the mean deviation for the participants.

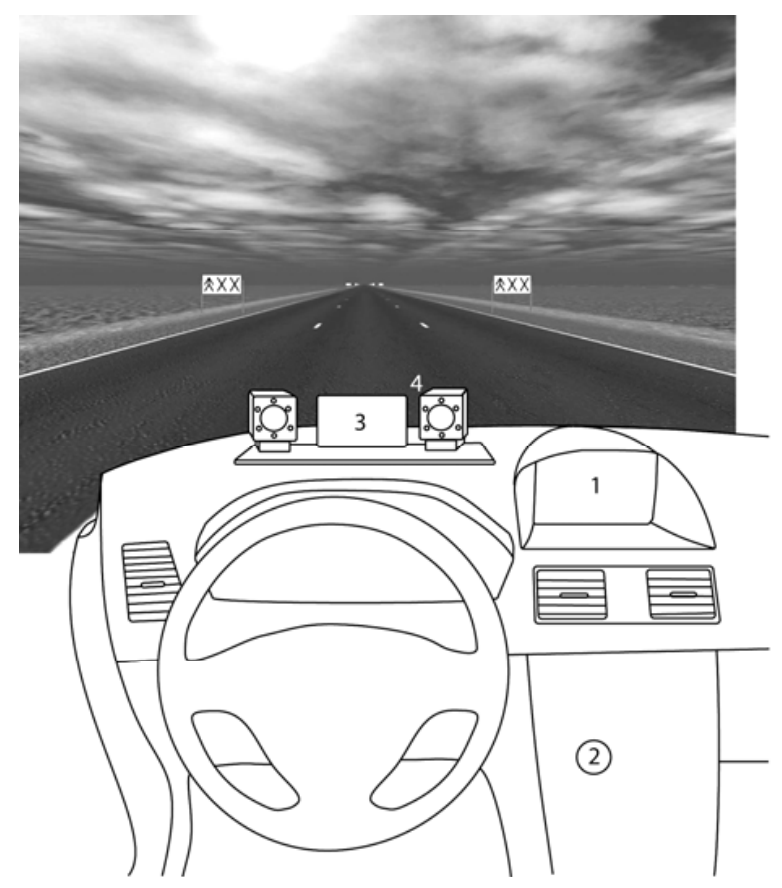

Figure 1. The LCT driving scene and Volvo XC90 simulator, including a secondary task interface display (1), a haptic rotary device (2), a display presenting the secondary tasks to be completed (3) and eye-tracking cameras (4).

The secondary task interface was implemented in Macromedia Director 8.5 (Adobe Systems Inc., USA). A visual menu was displayed on an $8.5^{\prime \prime}$ screen in the centre panel of the simulator (Fig. 1). The interaction with the interface was made with a haptic rotary device (ALPS Haptic Commander, ALPS Automotive Products Division, Japan) mounted on the centre panel. The device had a knob diameter of $3.5 \mathrm{~cm}$. The interface program managed the sensations provided through the rotary device and the graphical scenes displayed on the centre panel display. The program also managed the task presentation - orally in headphones and written on a 6.4" display placed on the dashboard in front of the participants (Fig. 1). faceLAB 4.1 (Seeing Machines, Australia) was used to record eye movements. By means of video signals from two cameras, the faceLAB system measures 3D head position and gaze direction at a rate of $60 \mathrm{~Hz}$. The two eye-tracking cameras were mounted on the dashboard in front of the participants (Fig. 1). To improve data quality face markers were placed on the participants' face. An analysis software, Visual Demand Measurement (VDM) Tool [28], was used to analyse the eye movement data. 


\subsection{Secondary Task Interface}

The secondary task interface was designed to contribute to the central theme of haptic and visual information and did not consist of real vehicle functions. The interface was designed to display congruent information in the haptic and visual modalities. Since textures can be effectively perceived both haptically and visually [6] a menu with texture items, or more specifically items with different roughness, was designed.

The four secondary task conditions were named: visual information $(\mathrm{V})$, visual information and haptic ridges $(\mathrm{VHr})$, visual information and haptic ridges and textures (VHrt), and haptic ridges and textures (Hrt). As the rotary device was turned in the conditions including visual information, a transparent blue cursor moved in the menu displayed in the centre panel display (Fig. 2). The four menu items, A, B, C and D, were arranged horizontally, and each texture had a height and width of $25 \mathrm{~mm}$. The graphical representations of the textures were identical for the three conditions including visual information. The visual menu was not displayed in the Hrt condition.



Figure 2. The visual menu displayed in the centre panel display in the secondary task conditions including visual information ( $\mathrm{V}, \mathrm{VHr}$ and VHrt). The textures are here presented in alphabetical order from $A$ to $D$. In the experiment, the active texture was marked with a transparent blue cursor.

The haptic sensations provided through the rotary device varied between the conditions (Fig. 3). The total angle of operation was $150^{\circ}$ for all conditions. Hence, a participant was able to comfortably rotate the device through the menu without changing the grasp. Restricting walls were incorporated outside the scale limits on each end of the menu, and a damper torque made forces increase and decrease with device speed. A smooth sensation was provided in the $\mathrm{V}$ condition as the device was turned. In the $\mathrm{VHr}$ condition salient ridges were incorporated between every texture in the menu to indicate borders. The angle of the ridges was $10^{\circ}$ and the amplitude of the elastic torque was $50 \mathrm{mN} \cdot \mathrm{m}$. The salient ridges indicated borders in the VHrt and Hrt conditions and, in addition, representations of the textures were provided through the device. The haptic textures were rendered as repeated and evenly distributed ridges, i.e. alternated high and low torque. The peak torque of the textures was $10 \mathrm{mN} \cdot \mathrm{m}$ and textures $\mathrm{A}, \mathrm{B}, \mathrm{C}$ and $\mathrm{D}$ had $0,3,6$ and 30 ridges, respectively.
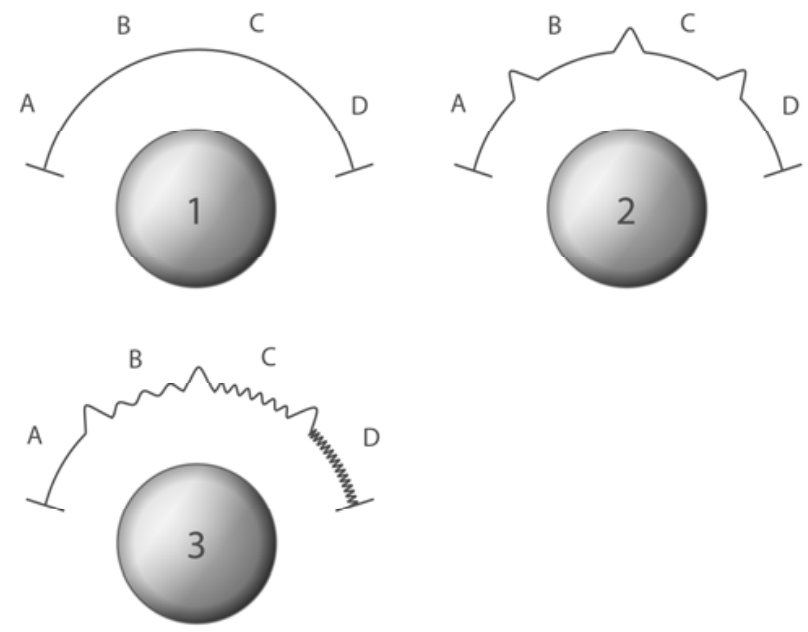
Figure 3. A representation of the haptic sensations provided through the rotary device in the four secondary task conditions: V (1), VHr (2), VHrt and Hrt (3).

\section{Task}

The tasks to be completed with the secondary task interface, e.g. "Locate C", were automatically presented to the participants orally in the headphones and provided in written form on the 6.4" display in front of the participants. The participants located and selected the requested item in the menu by turning and pushing the rotary device. As soon as one task was completed, the next was initiated. The target texture and the positions of the textures changed for every trial. If the wrong texture was selected, the textures stayed in the same order until the right texture was selected. A beep was given as feedback after a task was completed successfully. The device was programmed to start at the leftmost texture for every new trial, and the participants were to initially turn the device clockwise. The reset was not felt, and it was therefore not necessary to let go of the device.

\subsection{Procedure}

The experiment had a between-subjects design and the participants were randomly assigned to the four secondary task conditions, V, VHr, VHrt and Hrt. During the test the test leader sat in the front passenger seat of the simulator and controlled the equipment and read test instructions aloud from a manuscript. A brief description of the experiment as a whole was given at the beginning of a session. The participants were then instructed to adjust the seat, markers were placed on the participants' face, and the eye-tracking cameras were calibrated. The participants were given instructions about the LCT and were specifically informed to change lanes quickly, as soon as the information appeared on the signs. Each participant then drove three LCT tracks, of which the first two were training tracks and the third was a baseline driving track, i.e. driving without secondary task. Eye movements and driving performance were recorded for the baseline track.

Subsequent to baseline driving the participants practiced the secondary task in isolation in two training series. In the first series the participants were to learn which letter represented which 
texture and were free to explore a menu in which the menu items were provided in alphabetical order. As the rotary device was turned, the name of the active item (A, B, C or D) was displayed on the centre panel display. In the second training series the participants practiced the secondary task as it would be displayed while driving. The participants had to successfully complete 12 tasks in a row to pass the training.

Following this training the participants completed two dual-task driving tracks, i.e. driving with secondary task, of which the first was a training track. For the second track, data were recorded on secondary task performance, eye movements and driving performance. Since it may be tempting to adopt a strategy where secondary tasks are completed on the straight sections between the lane changes, the participants were instructed to perform as well as they could on both the primary and secondary tasks. The participants finally drove a second baseline track, for which data on eye movements and driving were recorded. After the test the participants were asked to fill in a participant characteristics form (concerning gender, age, handedness etc.) and mental workload (NASA-TLX) forms. A whole session took altogether about one hour.

\subsection{Dependent Measures}

Secondary task performance was measured in terms of the number of tasks completed and the number of push and turn errors made. When the wrong item was selected, the action was recorded as a push error, and the action was recorded as a turn error when the participants passed the right item without selecting. The eye movement data were analysed in terms of percent road centre (PRC) [29]. In the PRC analysis the road centre area was defined as a circle with a radius of $10^{\circ}$. LCT driving performance was measured in terms of mean deviation [14]. The NASA-TLX rating method was used to measure subjective mental workload. NASA-TLX is a multidimensional rating method that gives an overall workload score based on the weighted average of six workload-related factors (mental demand, physical demand, temporal demand, performance, effort and frustration level) [8].

\section{RRESULTS}

An alpha level of .05 was used for all statistical tests.

\subsection{Secondary Task Performance}

Figure 4 shows the number of tasks completed in the four secondary task conditions. A between-subjects ANOVA with secondary task condition ( $\mathrm{V}, \mathrm{VHr}$, VHrt and Hrt) as the factor was used to test the statistical significance of differences. The number of tasks completed was found to be significantly different between the conditions, $F(3,36)=21.1, p<.001$. The Tukey HSD procedure, used for post hoc pairwise comparisons of means, showed that there were no significant differences between the V, $\mathrm{VHr}$ and VHrt conditions. However, significantly fewer tasks were completed with the Hrt condition as compared to the other conditions (all $p<.001$ ).

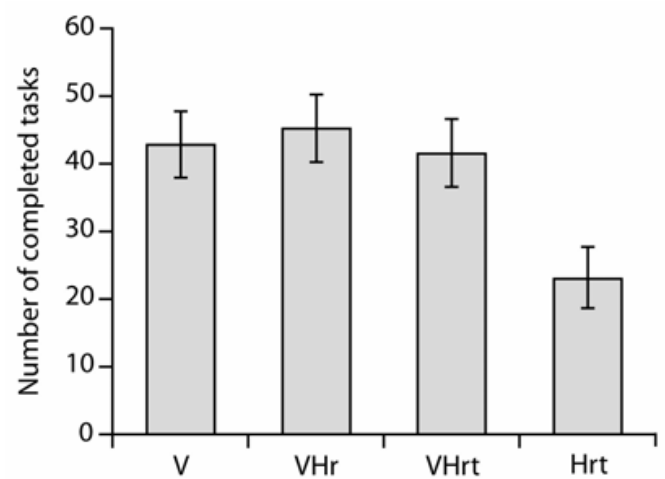

Figure 4. The number of tasks completed for the four secondary task conditions. The error bars represent $\mathbf{9 5 \%}$ confidence intervals for the means.

In terms of push error, floor effects were present for the $\mathrm{V}, \mathrm{VHr}$ and VHrt conditions, whereas $10 \%$ of the tasks in the Hrt condition included a push error. Figure 5 shows the percentage of tasks that included a turn error. To rectify the differences between group variances the turn error data was transformed by taking the square roots of the values. A between-subjects ANOVA with secondary task condition ( $\mathrm{V}, \mathrm{VHr}$, VHrt and Hrt) as the factor was used to test the statistical significance of differences. The number of turn errors made was found to be statistically different between the conditions, $F(3,36)=14.5, p<.001$. The Tukey HSD procedure showed that there were no significant differences between the V, VHr and VHrt conditions. However, significantly more turn errors were made with the Hrt condition as compared to the other conditions (all $p<.001$ ).

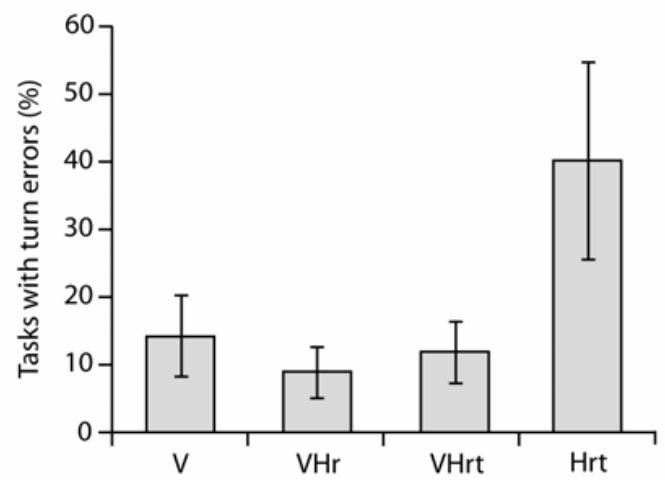

Figure 5. The percentage of tasks including a turn error in the four secondary task conditions. The error bars represent $95 \%$ confidence intervals for the means.

\subsection{Eye Movements}

Figure 6 shows the PRC values for baseline and dual-task driving for the four conditions. Since each participant conducted two baseline tracks, mean PRC values were calculated. Within-groups $t$-tests (two-tailed) were used to compare the PRC values between baseline and dual-task driving. The PRC values decreased drastically when tasks including visual information were performed: $t(9)=14.77, p<.001$, for the $\mathrm{V}$ condition, $t(9)=16.50$, 
$p<.001$, for the VHr condition and $t(9)=9.19, p<.001$, for the VHrt condition. No significant difference was found between baseline and dual-task driving for the Hrt condition.

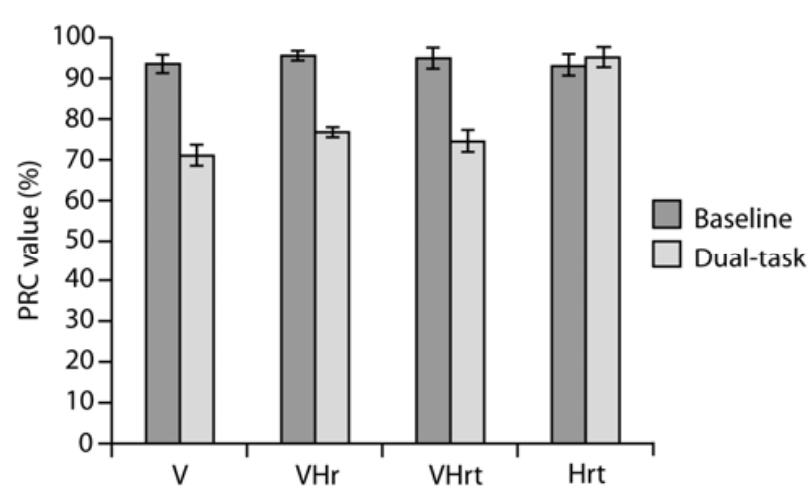

Figure 6. PRC values for baseline and dual-task driving for the four secondary task conditions. The error bars representing $95 \%$ confidence intervals for the means have been adjusted in the figure to suit within-subject comparisons [17].

To compare the PRC values between the conditions, the relative decrease or increase in PRC was calculated for each participant by dividing the dual-task value with the baseline value. More time spent looking at the centre area of the road in the dual-task condition as compared to the baseline leads to a higher quotient $(>1)$ (V: $M=0.62, S D=0.07$; VHr: $M=0.64, S D=0.07$; VHrt: $M=0.69, S D=0.10$; Hrt: $M=1.03, S D=0.06$ ). A betweensubjects ANOVA with secondary task condition (V, VHr, VHrt and Hrt) as the factor was used to test the statistical significance of differences. The quotient was found to be statistically different between the conditions, $F(3,36)=60.9, p<.0 .001$. The Tukey HSD procedure showed that there were no significant differences between the V, VHr and VHrt conditions. However, the time spent looking at the centre area of the road as compared to the baseline was significantly higher for the Hrt condition as compared to the other conditions (all $p<.001$ ).

\subsection{Driving Performance}

Figure 7 shows the mean deviation for baseline and dual-task driving tracks for the four conditions. Since each participant conducted two baseline tracks, mean baseline deviation values were calculated. Within-groups $t$-tests (two-tailed) were conducted to compare the mean deviation between baseline and dual-task driving. There was a significant increase in mean deviation from baseline driving to dual-task driving for all conditions: $t(9)=-3.1, p<.05$, for the $\mathrm{V}$ condition, $t(9)=-2.8$, $p<.05$, for the VHr condition, $t(9)=-2.8, p<.05$, for the VHrt condition and $t(9)=-5.06, p<.01$, for the Hrt condition.

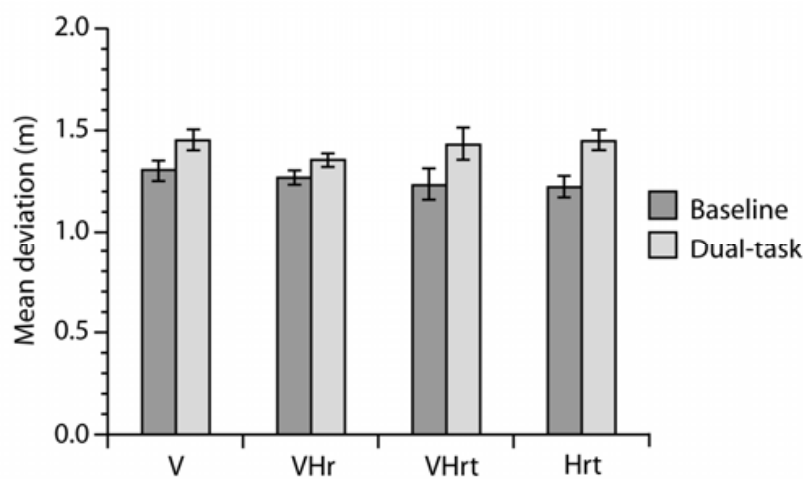

Figure 7. Mean deviations for baseline and dual-task driving for the four secondary task conditions. The error bars representing $\mathbf{9 5 \%}$ confidence intervals for the means have been adjusted in the figure to suit within-subject comparisons [17].

To compare the decrease in performance between the conditions, the relative decrease in performance was calculated for each participant by dividing the dual-task value with the baseline value (Mattes, 2003). Performing poorly in the dual-task condition as compared to the baseline leads to a higher quotient $(>1)(\mathrm{V}: M=$ $1.11, S D=0.12$; VHr: $M=1.06, S D=0.08$; VHrt: $M=1.16, S D$ $=0.18$; Hrt: $M=1.20, S D=0.13$ ). A between-subjects ANOVA with secondary task condition (V, VHr, VHrt and Hrt) as the factor was used to test the statistical significance of differences. Degradation in driving performance did not differ significantly between the conditions.

\subsection{Subjective Mental Workload}

The mean weighted workload scores (V: $M=58.3, S D=11.0$; VHr: $M=62.4, S D=14.7$; VHrt: $M=54.0, S D=18.9$; Hrt: $M=$ $62.9, S D=6.9$ ) were not significantly different between the secondary task conditions.

\section{DISCUSSION}

Fewer tasks were completed in the Hrt condition in comparison with the other three conditions. When using solely haptic information the menu had to be sequentially processed texture-bytexture, which was time-consuming, whereas the menu items in the $\mathrm{V}, \mathrm{VHr}$ and $\mathrm{VHrt}$ conditions could be visually processed more simultaneously. Analogously, more turn errors were made in the Hrt condition. A comparison between textures could be made visually without turning the device $(\mathrm{V}, \mathrm{VHr}$ and $\mathrm{VHrt})$, while the device had to be turned in order to compare textures haptically (Hrt), which resulted in an increased number of turn errors. Furthermore, and interestingly, it can be concluded that there were no significant differences between the three conditions including visual information, irrespectively of whether redundant haptic information was provided or not. Studies have shown that vision often dominates an integrated percept [5]. Thus, visual dominance in combination with the fact that the visual information in this experiment could be processed faster and more precise than the haptic seem to have led to a reliance on visual information when it was available. Rydström and Bengtsson [23] also observed in a desktop experiment that haptic texture 
information was often ignored by the participants if corresponding visual information was provided. Even though the present experiment included a concurrent visual task (driving) the participants do not seem to have used the redundant haptic information instead of or even as a complement to the visual. Nonetheless, in the domain of desktop interaction, augmenting a visual interface with usable haptic information through the interaction device has been shown to enhance the interaction [1, 4]. However, one difference in these interfaces is that the haptics is designed to enhance the visual interaction rather than replace it. Research in human-computer interaction has also shown that workload is decreased when a visual interface is augmented with usable haptic information [7, 20]. However, the reported mental workload did not differ between the secondary task conditions in this study. In view of the fact that the haptic interaction was more time consuming and less precise it is notable that it was not demonstrated that haptic interaction was more mentally demanding.

The PRC measure showed that the eyes were kept on the road during the non-visual Hrt condition. From this view, it is apparent that there is a potential for haptic interaction. In contrast, the PRC values were significantly lower for the $\mathrm{V}, \mathrm{VHr}$ and $\mathrm{Vhr}$ conditions compared to baseline driving. Further, in terms of the PRC values, the visual behaviour did not differ between the V, VHr and VHrt conditions. Hence, complementing visual information with corresponding haptic information did not increase the time the participants spent looking at the road. Interestingly, from a traffic safety point of view the non-visual haptic condition might be advantageous, even though it is more time consuming and less precise, as concluded above. A practical implication of this would be that when visual information is not needed it should not be provided. For example volume controls sometimes has a graphical representation in a display when the volume is increased or decreased. The visual information may make the driver look at the display instead of only rely on the auditory and haptic information.

When using the LCT, which is a PC simulated environment, the road scene is projected only in front of the participants, and no rear-view mirrors are used. In addition, no glances at the speedometer are necessary since the driving speed is systemcontrolled. In this experiment there were therefore relatively high values in the PRC value for baseline driving, over 90\%. Real driving normally gives a PRC value of about $70 \%$ [29]. This may have made it infeasible to calculate an effect of gaze concentration caused by attention to a non-visual secondary task $[22,29]$. It should also be mentioned that glances at the display presenting the tasks to be completed may have induced some noise in the data.

Compared to baseline driving, all four secondary task conditions caused an increase in mean deviation. This finding supports research showing that both visual and non-visual secondary tasks have a negative influence on driving performance $[15,33]$. Furthermore, it was shown that the participants performed similarly in all conditions. It could, however, perhaps be expected that the degradation in driving performance should be less for the Hrt condition since the eyes were kept on the road. One possible explanation for the absence of this effect could be that the lanechange signs in the LCT are highly expected. A participant can therefore adopt a strategy in which the tasks are solved using visual information at the straight sections between the signs. Alternatively, since the tasks were provided one after another during the dual-task track, most of the driving was spent with only one hand on the steering wheel. In view of the fact that LCT requires a great deal of manipulation of the steering wheel, and large corrections of the steering wheel often actually require the use of both hands [31], there was substantial manual time-sharing for all conditions. However, since the LCT derives a single measure of driving performance, different characteristics of driving are not considered in isolation. Perhaps other measures, such as response to an unexpected external event, could capture any differences.

Porter et al. [21] found that an in-car interface designed with consideration to haptics was preferable to a conventional one in terms of the number and duration of eye glances. The study of Porter et al. indicates that haptic interaction has the potential to facilitate the interaction with in-vehicle equipment. The present study showed that during the condition including only haptic information the participants' eyes remained on the road during the interaction. Even if the experimental task took longer when using only haptic information, the degradation in performance and mental workload assessment did not differ from the conditions including visual information, which is a result of vital importance for future implementations of haptics. However, the haptic information needs to be improved. Multifunctional, menu-based systems are common in cars today [24]. These systems include a wide range of different functions, and the interaction often requires several steps of visual and manual interaction. This experiment serves as a basis for investigating the use of haptic and visual information in the interaction with such systems. The next step will be to implement haptic and visual information in combination with a more genuine in-car interface.

\section{ACKNOWLEDGMENTS}

This study was supported by the Swedish Program Board for Automotive Research.

\section{REFERENCES}

[1] Akamatsu, M., MacKenzie, I.S., 1996. Movement characteristics using a mouse with tactile and force feedback. Int. J. Human-Computer Studies 45, 483-493.

[2] Bengtsson, P., Grane, C., Isaksson, J., 2003. Haptic/graphic interface for in-vehicle comfort functions - a simulator study and an experimental study, in: Proc. of the IEEE International Workshop on Haptic, Audio and Visual Environments and Their Applications. Ottawa, Canada, pp. 25-29.

[3] Burnett, G.E., Porter J.M., 2001. Ubiquitous computing within cars: designing controls for non-visual use. Int. J. Human-Computer Studies 55, 521-531.

[4] Campbell, S.C., Shumin, Z., May, K.W., Maglio, P.P., 1999. What you feel must be what you see: adding tactile feedback to the Trackpoint, in: Sasse, M.A., Johnson, C. (Eds.), Human-Computer Interaction - Proceedings of INTERACT'99. IOS Press, Edinburgh, pp. 383-390.

[5] Ernst, M.O., Bülthoff, H.H., 2004. Merging the senses into a robust percept. Trends. Cogn. Sci. 4, 162-169. 
[6] Gentaz, E., Hatwell, Y., 2003. Haptic processing of spatial and material object properties, in: Hatwell, Y., Strieri, A., Gentaz, E. (Eds.), Touching for Knowing: Cognitive Psychology of Haptic Manual Perception. J. Benjamins, Amsterdam, pp. 123-159.

[7] Grane, C., Bengtsson, P., 2005. Menu selection with a rotary device founded on haptic and/or graphic information, in: Proc. of World Haptics 2005. Piza, Italy, pp. 475-476.

[8] Hart, S.G., Staveland, L.E., 1988. Development of NASATLX (Task Load Index): results of empirical and theoretical research, in: Hancock, P.A., Meshkati, N. (Eds.), Human Mental Workload. Elsevier, Amsterdam, pp. 139-182.

[9] Hatwell, Y., 2003. Introduction, in: Hatwell, Y., Strieri, A., Gentaz, E. (Eds.), Touching for Knowing: Cognitive Psychology of Haptic Manual Perception. J. Benjamins, Amsterdam, pp. 1-14.

[10] Hayward, V., Astley, O.R., Cruz-Hernandez, M., Grant, D., Robles-De-La-Torre, G., 2004. Haptic interfaces and devices. Sensor Review 24, 16-29.

[11] Ho, C., Tan, H.Z., Spence, C., 2005. Using spatial vibrotactile cues to direct visual attention in driving scenes. Transport. Res. F 8, 397-412.

[12] Hollis, R., 2004. Haptics, in: Bainbridge, W.S. (Ed.), Berkshire Encyclopedia of Human-Computer Interaction. Berkshire Publishing Group, Great Barrington, pp. 311-316.

[13] ISO 15007-1. Road vehicles - Measurement of driver visual behaviour with respect to transport information and control systems - Part 1: Definitions and parameters.

[14] ISO 26022 (standard under development). Road vehicles Ergonomic aspects of transport information and control systems - Simulated lane change test to assess driver distraction.

[15] Lamble, D., Kauranen, T., Laakso, M., Summala, H., 1999. Cognitive load and detection thresholds in car following situations: safety implications for using mobile (cellular) telephones while driving. Accid. Anal. Prev. 31, 617-623.

[16] Lee, J.D., Hoffman, J.D., Hayes, E., 2004. Collision warning design to mitigate driver distraction, in: Proc. of CHI 2004. ACM Press, New York, pp. 65-72.

[17] Loftus, G. R., Masson, M. E., 1994. Using confidence intervals in within-subject designs. Psychon. B. Rev. 1, 476490.

[18] Mattes, S., 2003. The lane-change-task as a tool for driver distraction evaluation, in: Strasser, H., Kluth, K., Rausch, H., Bubb, H. (Eds.), Quality of Work and Products in Enterprises of the Future. Ergonomia Verlag, Stuttgart, pp. 57-60.

[19] Moore, 1974. Tactile and kinaesthetic aspects of pushbuttons. Appl. Ergon. 5, 66-71.
[20] Oakley, I., McGee, M. R., Brewster, S., Gray, P., 2000. Putting the feel in 'look and feel', in: Proc. of CHI 2000. ACM Press, New York, pp. 415-422.

[21] Porter, J.M., Summerskill, S.J., Burnett, G.E., Prynne, K., 2005. BIONIC - 'eyes-free' design of secondary driving controls, in: Proc. of the Accessible Design in the Digital World Conference. Dundee, UK.

[22] Recarte, M.A, Nunes, L.M., 2003. Mental workload while driving: effects of visual search, discrimination, and decision making. J. Exp. Psychol. Appl. 9, 119-137.

[23] Rydström, A., Bengtsson, P., 2007. Haptic, visual and crossmodal perception of interface information, in: de Waard, D., Hockey, G.R.J., Nickel, P., Brookhuis, K.A. (Eds.), Human Factors Issues in Complex System Performance. Shaker Publishing, Maastricht, pp. 399-409.

[24] Rydström, A., Bengtsson, P., Grane, C., Broström, R., Agardh, J, Nilsson, J., 2005. Multifunctional systems in vehicles: a usability evaluation, in: Thatcher, A., James, J., Todd A. (Eds.), Proceedings of CybErg 2005, the Fourth International Cyberspace Conference on Ergonomics. International Ergonomics Association Press: Johannesburg.

[25] Sivak, M., 1996. The information that drivers use: is it indeed 90\% visual? Perception 25, 1081-1089.

[26] Strayer, D.L., Johnston, E.A., 2001. Driven to distraction: dual-task studies of simulated driving and conversing on a cellular phone. Psychol. Sci. 12, 462-466.

[27] Van Erp, J., Van Veen, H., 2004. Vibrotactile in-vehicle navigation system. Transport. Res. F 7, 247-256.

[28] Victor, T.W., Blomberg, O., Zelinsky, A., in press. Automating driver visual behavior measurement. Vision in Vehicles 9.

[29] Victor, T.W., Harbluk, J.L., Engström, J.A., 2005. Sensitivity of eye-movement measures to in-vehicle task difficulty. Transport. Res. F 8, 167-190.

[30] Vilimek, R., Hempel, T., Otto, B., 2007. Multimodal interfaces for in-vehicle applications. Lect. Notes. Comput. Sc. 4552, 216-224.

[31] Wierwille, W., 1993. Demands on driver resources associated with introducing advanced technology into the vehicle. Transport. Res. C 1, 133-142.

[32] Wikman, A.S., Nieminen, T., Summala, H., 1998. Driving experience and time-sharing during in-car tasks on roads of different width. Ergonomics 41, 358-372.

[33] Zwahlen, H.T., Adams, C.C., Jr., DeBald, D.P., 1988. Safety aspects of CRT touch panel controls in automobiles, in: Gale, A.G., Freeman, M.H., Haslegrave, C.M., Smith, P.A., Taylor, S.P. (Eds.), Vision in Vehicles II. Elsevier, Amsterdam, pp. 335-344. 